\title{
TERAPIA NUTRICIONAL NO PACIENTE CRITICAMENTE ENFERMO
}

\author{
NUTRITIONAL THERAPEUTIC FOR THE CRITICALLYILL PATIENTS
}

Jussara Fialho Ferreira Côrtes ${ }^{1}$; Sandra Lúcia Fernandes'; Isolda Prado de Negreiros Nogueira-Maduro²; Anibal Basile Filho ${ }^{3}$; Vivian M. M. Suen; José Ernesto dos Santos ${ }^{4}$; Hélio Vannucchi ${ }^{4}$ Júlio Sérgio Marchini ${ }^{4}$.

${ }^{1}$ Médica residente da Divisão de Nutrologia do Departamento de Clínica Médica. ${ }^{2}$ Médica nutróloga, Pós-graduanda. ${ }^{3}$ Docente da Disciplina de Terapia Intensiva do Departamento de Cirurgia e Anatomia. ${ }^{4}$ Docentes da Divisão de Nutrologia do Departamento de Clínica Médica. Faculdade de Medicina de Ribeirão Preto - USP.

CorRespondêncIA: Jussara Fialho Ferreira Côrtes. Unidade Metabólica. Divisão de Nutrologia. Departamento de Clínica Médica. Av. Bandeirantes, 3900 - 5aandar. CEP 14049-900 - Ribeirão Preto - SP. E-mail: jussffcortes@hotmail.com / jsmarchini@fmrp.usp.br

CORTES JFF; FERNANDES SL; NOGUEIRA-MADURO IPN; BASILE-FILHO A; SUEN VMM; SANTOS JE; VANNUCHI H \& MARCHINI JS. Terapia nutricional no paciente criticamente enfermo. Medicina, Ribeirão Preto, 36: 394-398, abr./dez.2003.

RESUMO - A Terapia Nutricional tem tido grande impacto na evolução do paciente grave. Sua escolha e manuseio, no entanto, têm sido alvo de dúvidas e erros freqüentes, principalmente no paciente crítico. A escolha da terapia nutricional a ser realizada, bem como sua via de administração deve levar em conta o quadro clínico e as condições gerais do paciente. A via oral/enteral de oferta de nutrientes, mais fisiológica, é a preferível. Portanto, sempre que possível, deve-se utilizar o trato gastrointestinal. Quando este não puder ser utilizado, a via parenteral está indicada, tanto no sentido de suplementar a nutrição enteral, como quando esta não consegue suprir toda a demanda de nutrientes, de que o paciente necessita. Esta revisão tem por objetivo apresentar e difundir a condução da terapia nutricional no paciente crítico, de forma prática e adequada.

De maneira geral, a oferta calórica deverá atender às necessidades basais do paciente e a protéica fornecer material plástico para síntese protéica. Minerais, vitaminas e água deverão ser ajustados às necessidades e ao quadro clínico do paciente em questão.

UNITERMOS - Avaliação Nutricional. Estado Nutricional. Apoio Nutricional. Nutrição Enteral. Nutrição Parenteral. Estado Terminal.

\section{1- INTRODUÇÃO}

Denomina-se terapia nutricional a oferta de nutrientes pelas vias oral, enteral e/ou parenteral, visando à oferta terapêutica de proteínas, energia, minerais, vitaminas e água, adequadas aos pacientes, que, por algum motivo, não possam receber suas necessidades pela via oral, convencional. Paralelamente, a desnutrição hospitalar é uma situação freqüente, conhecida como predisponente para um aumento na morbidade $\mathrm{e}$ mortalidade, resultando em hospitalização prolongada e onerosa ${ }^{(1)} \mathrm{O}$ paciente, em terapia intensiva, freqüentemente, encontra-se em estado hipermetabólico, decorrente do trauma, sepse ou de qualquer outro quadro de gravidade. O suporte nutricional para tais pacientes pode ser decisivo em sua evolução.

Esta revisão tem a finalidade de expor as situações em que a terapia nutricional para os pacientes em estado grave está indicada, bem como as quantidades de nutrientes a serem prescritas. 


\section{2- FISIOPATOLOGIA}

A prescrição adequada da terapia nutricional ao paciente grave depende do entendimento da resposta metabólica que se segue à agressão aguda - trauma, choque, sepse e inflamação sistêmica. Tal situação caracteriza-se por uma resposta generalizada, em que há mobilização de energia para estimular a função imune e o reparo dos tecidos lesados, o que ocorre às custas do consumo de massa magra e aumento na perda urinária, de nitrogênio. Os aminoácidos são mobilizados do músculo esquelético, tecido conjuntivo e intestino, para promover a cicatrização das feridas e para a síntese hepática de proteínas de fase aguda, além de tornarem-se substrato para a gliconeogênese. ${ }^{(2,3)}$

A síntese de albumina diminui, compensando a maior síntese de substâncias nitrogenadas, denominadas proteínas de fase aguda, entre elas: proteína $\mathrm{C}$ reativa, mucoproteínas, fibrinogênio, transferrina, ceruloplasmina, e fatores do complemento. O papel desses elementos encontra-se apenas parcialmente elucidado, mas acredita-se que, em sua maioria, tenham a função de amenizar as repercussões da invasão bacteriana, do choque e das lesões teciduais. Diversas proteínas da fase aguda comportam-se como antiproteases e opsoninas, outras auxiliam na coagulação sanguínea e algumas parecem contribuir com a cicatrização das feridas. ${ }^{(1)}$

\section{3- INDICAÇÃ̃O}

A terapia nutricional não somente procura prevenir a deterioração do estado nutricional, como, também é efetiva para minimizar as complicações devido ao jejum prolongado. Nessa condição, a atrofia da mucosa intestinal contribui para o aumento da permeabilidade e translocação bacteriana da luz para os linfonodos mesentéricos. A perda ponderal, excedendo $10 \%$ do peso, antes da doença, pode comprometer a habilidade do paciente em combater infecções e, em tais casos, a terapia nutricional está indicada. ${ }^{(4,5,6)}$

A identificação da desnutrição proteicocalórica é feita por meio da história clínica / exame físico, perda ponderal, medidas antropométricas [peso, altura, comprimento do braço ( $\mathrm{Cob})$, circunferência média do braço $(\mathrm{Cb})$ e prega tricipital $(\mathrm{Pt})]$, índice de massa corporal (peso/altura ${ }^{2}=\mathrm{kg} / \mathrm{m}^{2}$ ), circunferência muscular do braço $[\mathrm{Cb}(\mathrm{cm})-\pi \times \mathrm{Pt}(\mathrm{cm})]$, reserva de gordura do braço $\left[\mathrm{Pt} / \mathrm{Cob}^{2}=\mathrm{mm} / \mathrm{dm}^{2}\right]$, concentrações séricas de albumina / transferrina e índice nutricional de risco. Os pacientes tornam-se nutricionalmente de risco, quando a desnutrição resulta em aumento da morbidade específica. ${ }^{(7)}$ São múltiplos os fatores de risco para desnutrição, como podem ser vistos na Tabela I.

Tabela I - Fatores de riscos que predispõem à desnutrição protéica e calórica

- Problemas clínicos:

Cirurgia recente ou trauma

Sepse

Doença crônica

Efeitos de radio ou quimioterapia

- Distúrbios gastrointestinais:

Anorexia, outros transtornos alimentares; disfagia; náusea recorrente, vômitos, ou diarréia profusa Pancreatite, doença inflamatória intestinal, fístulas gastrointestinais

Síndrome do intestino curto

- Problemas psicossociais:

Alcoolismo

Uso de drogas

Pobreza

Isolamento

- Dietas anormais:

Dieta restrita

Recente diminuição na ingestão alimentar

\section{4- AVALIAÇÃO NUTRICIONAL}

Os dados antropométricos podem estar alterados devido ao excesso de água corpórea, secundário, por exemplo, à hipoalbuminenia. A oferta calórica é calculada em relação ao valor estimado para o metabolismo basal (GEB), considerando-se o peso atual do paciente sem acrescentar fatores de correção ao valor encontrado. ${ }^{(1)}$ Idealmente, seria indicada a medida real do gasto calórico por meio de calorimetria indireta (determinação do $\mathrm{Vo}_{2}$ e $\mathrm{Vco}_{2}$ ). Uma das maneiras de estimar o metabolismo basal é por meio da equação de Harris - Benedict. ${ }^{(8)}$

- Para mulheres, GEB $(\mathrm{kcal} / \mathrm{dia})=655,1+9,6 \times$ Peso $(\mathrm{em} \mathrm{kg})+1,85$ x Altura (em cm) -4,68x Idade (em anos).

- Para homens, GEB (kcal/dia) $=66,47+13,75 \times$ Peso $(\mathrm{em} \mathrm{kg})+5 \times$ Altura $(\mathrm{em} \mathrm{cm})-6,76 \mathrm{x}$ Idade (em anos).

Em pacientes que não respondem a cálculos estimados, pacientes com insuficiência de múltiplos 
órgãos, com suporte nutricional prolongado, ou pacientes com insuficiência respiratória, aguda, em ventilação artificial, recomenda-se o uso de calorimetria indireta. Nessa situação, o GEB é igual a 3,9 x Vo $\mathrm{Vo}_{2}+$ $1,1 \times \mathrm{Vco}_{2}$.

\section{5- PRESCRIÇÃO NUTRICIONAL NO PACI- ENTE GRAVE ${ }^{(7)}$}

Como conduta geral, deve-se instituir terapia nutricional, se:

- paciente sem nutrição há sete dias com índice de massa corporal (IMC) $>18 \mathrm{~kg} / \mathrm{m}^{2}$;

- paciente sem nutrição há mais de três dias, se IMC $\leq 18 \mathrm{~kg} / \mathrm{m}^{2}$;

- estimativa da duração da doença, que impossibilita a ingestão, via oral, de alimentos, acima de 10 dias;

- pacientes com perda ponderal, aguda, maior que 10\%;
- pacientes de alto risco - com infecção grave, queimado, com traumatismo grave.

Em todas essas situações, iniciar terapia nutricional após estabilização hemodinâmica do paciente.

Se o trato gastrointestinal (TGI) estiver funcionante, fornecer nutrição enteral; usar sonda em posição gástrica, se o risco de aspiração for baixo; caso contrário, usar sonda em posição jejunal. Sempre monitorizar a presença de resíduos gástricos, observando o surgimento de distensão abdominal, diarréia e anormalidades hidroeletrolíticas. Se TGI não estiver funcionante (íleo paralítico, obstrução intestinal, hemorragia gastrointestinal), iniciar NPT via cateter central. Nessa situação, monitorizar a glicemia, distúrbios hidroeletrolíticos e acidobásicos além das condições gerais do paciente. Alguns pacientes com TGI funcionante não irão ou não conseguirão comer. São aque-

Tabela II - Sugestão de Cálculo de Nutrição Parenteral. ${ }^{(14)}$

\begin{tabular}{|c|c|c|c|}
\hline Passos & Considerações clínicas & Variação-padrão & $\begin{array}{l}\text { Exemplo para adulto de } 40 \text { anos, } \\
\text { com } 70 \mathrm{~kg} \mathrm{e} 1,80 \mathrm{~m} \text {. }\end{array}$ \\
\hline $\begin{array}{l}\text { 1. Estimar o gasto } \\
\text { energético total } \\
\text { (GEB) kcal/ } 24 \mathrm{~h}\end{array}$ & & & $\mathrm{GEB} \cong 1700 \mathrm{Kcal}$ \\
\hline $\begin{array}{l}\text { 2. Estebelecer a ne- } \\
\text { cessidade protéica. } \\
\text { Proteína = } 4 \mathrm{kcal} / \mathrm{g}\end{array}$ & $\begin{array}{l}\text { Insuficiência renal = } \\
0,6 \mathrm{~g} / \mathrm{kg} / \text { dia; aumentar para } \\
1-1,2 \mathrm{~g} / \mathrm{kg} / \text { dia, se em diálise. } \\
\text { Encefalopatia hepática = } \\
0,6 \mathrm{~g} / \mathrm{Kg} / \text { dia. } \\
\text { Hepatopatia crônica }= \\
1-1,2 \mathrm{~g} / \mathrm{kg} / \text { dia. }\end{array}$ & $\begin{array}{l}0,8 \text { a } 2,0 \mathrm{~g} / \mathrm{kg} / \mathrm{dia} \\
\text { desconsiderar a oferta } \\
\text { calórica da proteína, } \\
\text { pois a mesma, ideal- } \\
\text { mente, tem função } \\
\text { plástica. }\end{array}$ & $\begin{array}{l}1,0 \mathrm{~g} / \mathrm{kg} \\
1,0 \times 70 \mathrm{Kg} \\
70 \mathrm{~g} / 24 \mathrm{~h}\end{array}$ \\
\hline $\begin{array}{l}\text { 3. Verificar o conteú- } \\
\text { do de carboidrato da } \\
\text { fórmula. } \\
\text { Glicose } 1 \mathrm{~g}=4 \mathrm{kcal}\end{array}$ & $\begin{array}{l}\text { Baseado na tolerância de } \\
\text { glicose mais do que na } \\
\text { gravidade da doença. }\end{array}$ & $\begin{array}{l}70-90 \% \text { do GEB } \\
* \text { Vig : até } 5 \mathrm{mg} / \mathrm{Kg} / \mathrm{min} \\
\text { (Velocidade de infusão } \\
\text { da glicose) }\end{array}$ & $\begin{array}{l}70 \% \text { do GEB. } \\
1700 \mathrm{kcal} \times 70 \%=1190 \mathrm{kcal} / \mathrm{dia} \\
1190 \mathrm{kcal} \div 4 \mathrm{kcal}=298 \mathrm{~g} / 24 \mathrm{~h} \\
\mathrm{Vig}=298000 \mathrm{mg} \div 70 \div 1140 \cong \\
3,7 \mathrm{mg} / \mathrm{Kg} / \mathrm{min}\end{array}$ \\
\hline $\begin{array}{l}\text { 4. Cálculo do } \\
\text { conteúdo lipídico. }\end{array}$ & $\begin{array}{l}\text { A dose não deve exceder } \\
1 \mathrm{~g} / \mathrm{kg} / \mathrm{dia}\end{array}$ & $10-30 \%$ do GEB & $\begin{array}{l}30 \% \text { do GEB }=510 \mathrm{kcal} \\
510 \mathrm{kcal} \div 9 \mathrm{kcal}=57 \mathrm{~g} / 24 \mathrm{~h} \\
57 \mathrm{~g} \div 70 \mathrm{~kg} \cong 0,8 \mathrm{~g} / \mathrm{kg} / \mathrm{dia}\end{array}$ \\
\hline $\begin{array}{l}\text { 5. Calcular } \\
\text { necessidades hídricas }\end{array}$ & $\begin{array}{l}\text { Varia com estado clínico, } \\
\text { função orgânica, atividade, e } \\
\text { perdas do trato gastrointestinal }\end{array}$ & $30-40 \mathrm{ml} / \mathrm{kg} / \mathrm{dia}$ & $30 \times 70=2100 \mathrm{ml} / 24 \mathrm{~h}$ \\
\hline $\begin{array}{l}\text { 6.Determinar aditivos } \\
\text { essenciais (eletrólitos } \\
\text { e vitaminas) }\end{array}$ & $\begin{array}{l}\text { Nos casos de nutrição pa- } \\
\text { renteral exclusiva, suple- } \\
\text { mentar vitamina K. }\end{array}$ & Segundo Tabela III & \\
\hline
\end{tabular}


les com obstrução orofaríngea ou esofagiana e ainda os pacientes portadores de seqüela neurológica, para os quais a alimentação nasogástrica ou nasojejunal pode estar indicada. ${ }^{(7)}$

A via utilizada para terapêutica nutricional deverá, sempre que possível, ser enteral, em virtude de ser mais fisiológica e de menor custo.

A Nutrição Parenteral (NP) é uma solução ou emulsão, contendo carboidratos, aminoácidos, lipídios, água, vitaminas e minerais, administrada por via intravenosa, com a finalidade de fornecer aos pacientes os nutrientes em quantidades necessárias para a síntese e/ou a manutenção dos tecidos, órgãos ou sistemas. ${ }^{(9)}$ É indicada, quando houver empecilho na alimentação via oral ou enteral ou, ainda, quando a absorção dos nutrientes for incompleta ou insuficiente. Planejar NP é um processo que deve ser seguido passo a passo (Tabela II) levando-se em conta as necessidades energéticas, protéicas, vitamínicas e eletrolíticas. O primeiro passo é definir, por meio dos cálculos expostos, metas para a oferta energética, e distribuir as calorias entre carboidratos, proteínas e lipídios.

Em NP, as recomendações de proteínas, no paciente em estado grave, têm sido discutidas, sugerindo de $1,0-2,0 \mathrm{~g} / \mathrm{kg} / \mathrm{dia}$, de acordo com a condição clínica e o estado catabólico ${ }^{(7)}$. As recomendações de lipídios são de 1-2 g/kg/dia. Quando a solução de lipídios não puder ser adicionada à NP na fórmula 3:1 (glicose; aminoácidos e lipídios na mesma solução) os lipídios devem ser ofertados 2-3x/semana, para fornecer ácidos graxos essenciais, prevenindo sua deficiência. A maior parte das calorias, em geral, é obtida com oferta de glicose. Para evitar, principalmente, a hiperglicemia e a sobrecarga pulmonar pelo excesso de produção de $\mathrm{CO}_{2}$, é recomendado manter a taxa de infusão de glicose em, no máximo, $5 \mathrm{mg} / \mathrm{kg} / \mathrm{min}$ (Vig-velocidade de infusão da glicose).

Em virtude de a maioria dos pacientes que recebem NP apresentarem quadro de má absorção e grande perda hidroeletrolítica, podem ocorrer alterações de eletrólitos e minerais como $\mathrm{Na}^{+}, \mathrm{K}^{+}, \mathrm{Ca}^{++}$, $\mathrm{Mg}^{++}, \mathrm{PO}_{4}^{-}, \mathrm{Cl}^{-}$. A necessidade diária de minerais e vitaminas para pacientes em bom estado cardiovascular, intestinal, renal e hormonal é apresentada na Tabela III. Em caso de perdas excessivas ou retenção anormal pelo intestino ou rins, prescrições precisam ser individuais, com monitorização constante. ${ }^{(7)}$

As soluções de NP devem ser mantidas em recipientes apropriados e em temperatura entre $2^{\circ} \mathrm{e}$ $8^{\circ} \mathrm{C}$, durante o armazenamento e/ou transporte por um período máximo de 24 h e sua administração deve ser realizada, preferencialmente, com bomba de infusão à temperatura ambiente. ${ }^{(9)}$ Cada instituição define soluções-padrão que têm, como vantagem, a estabilidade, menor custo e menor manipulação no preparo, embora, às vezes, não atendam às necessidades individuais. Já com as preparações individualizadas, ocorre o inverso. ${ }^{(10,11)}$

É importante conhecer as situações especiais em nutrição do paciente criticamente enfermo, como por exemplo: seu tempo de utilização; em pacientes com insuficiência respiratória ou, ainda, nos pacientes com insuficiência renal, cuja oferta protéica varia de acordo com o fato de o paciente estar ou não em diálise, deve ser evitada a oferta excessiva de hidratos de carbono, que resulta em aumento da produção de $\mathrm{CO}_{2} \cdot{ }^{(12,13)}$

Tabela III - Recomendação diária de nutrientes, minerais e vitaminas

\begin{tabular}{|c|c|}
\hline Nutrientes & Unidade \\
\hline Cálcio & $0,2-0,4 \mathrm{~g}$ \\
Fósforo & $0,4-0,8 \mathrm{~g}$ \\
Potássio & $3-4 \mathrm{~g}$ \\
Sódio & $1-3 \mathrm{~g}$ \\
Cloro & $3-4 \mathrm{~g}$ \\
Magnésio & $0,3 \mathrm{~g}$ \\
Ferro & $1-2 \mathrm{mg}$ \\
Zinco & $3-12 \mathrm{mg}$ \\
Cobre & $0,3-0,5 \mathrm{mg}$ \\
\hline Iodo & $0,15 \mathrm{mg}$ \\
Manganês & $2-5 \mathrm{mg}$ \\
Cromo & $15-30 \mu \mathrm{g}$ \\
\hline Molibidênio & $20-120 \mu \mathrm{g}$ \\
\hline Selênio & $50-100 \mu \mathrm{g}$ \\
\hline Ácido ascórbico & $100 \mathrm{mg}$ \\
Tiamina & $3 \mathrm{mg}$ \\
\hline Riboflavina & $3,6 \mathrm{mg}$ \\
Niaciana & $40 \mathrm{mg}$ \\
Biotina & $60 \mu \mathrm{g}$ \\
\hline Ácido pantotênico & $15 \mathrm{mg}$ \\
Piridoxina & $4 \mathrm{mg}$ \\
\hline Ácido fólico & $400 \mu \mathrm{g}$ \\
Cobalamina & $5 \mu \mathrm{g}$ \\
Vitamina A & $1 \mathrm{mg}$ \\
Vitamina D & $5-10 \mu \mathrm{g}$ \\
Vitamina E & $10-15 \mathrm{mg}$ \\
Vitamina K & $200 \mu \mathrm{g}$ \\
\hline
\end{tabular}




\section{6- CONCLUSÃO}

O tratamento do paciente grave deve incluir a terapia nutricional específica, uma vez que são pacientes com alto risco de complicações. Após estratificados os riscos, deve-se optar pela melhor via de terapia nutricional. A terapêutica nutricional, no paciente criticamente enfermo, pode resultar numa melhor evolução da doença de base com maior sobrevida e menor ônus hospitalar. Vale a pena ressaltar que a via oral é sempre a preferível, e que a indicação da nutrição parenteral não deve ser retardada nos casos apropriados.

CORTES JFF; FERNANDES SL; NOGUEIRA-MADURO IPN; BASILE-FILHOA; SUEN VMM; SANTOS JE; VANNUCHI H \& MARCHINI JS. Nutritional therapeutic for the critically ill patients. Medicina, Ribeirão Preto, 36: 394-398, apr./dec. 2003.

ABSTRACT - Nutritional Therapeutic has a great impact on the treatment of the critically ill patient. Its choice and conduct, however, has been a matter of doubt and frequent errors.

The choice of nutritional therapeutic and its route of administration must consider if the gastrointestinal tract is available. The enteral route will be used if the gastrointestinal tract is available. If it isn't; like short bowel syndrome, or gastrointestinal haemorrhage, parenteral nutrition is indicated.

This review has the objective of answer the most frequent questions and conduct the nutritional therapeutic in critically ill patients.

UNITERMS - Nutrition Assessment. Nutritional Status. Nutritional Support. Enteral Nutrition. Parenteral Nutrition. Critical Ilness.

\section{REFERÊNCIAS BIBLIOGRÁFICAS}

1 - POWELL-TUCK, J. Nutrition. Ball Clin Anaesthesiol 13: 411-425,1999.

2 - BARTON RG. Nutrition support in critical illness. Nutr Clin Pract 9: 127-139, 1994.

3 - KINNEY M. Metabolic responses of critically ill pacients. Crit Care Clin 11: 569-585,1995.

4 - KUDSK KA. Clinical applications of enteral nutrition. Nutr Clin Pract 9:165-171,1994.

5 - BARTON RG. Immune-enhancing enteral formulas: are they beneficial in critical pacients? Nutr Clin Pract 12:51-62,1997.

6 - SOUBA WW; KLIMBERG VS; PLUMLEY DA; SALLOUM RM; FLLYNNTC; BAND KI \& COPELAND EM 3rd. The role of glutamin in mataining a healthy gut and supportingthe metabolic response to injury and infection. J Surg Res 48 :383391,1990 .

7 - SOUBA WW \& WILMORE D. Diet and nutrition in the care of the pacient with surgery, trauma and sepsis.In:SHILS ME; OLSON JA; SHIKE M \& ROSS AC, eds. Modern nutrition in health and disease, 9th ed, Lippincott Williams \& Wilkins, Pennsylvania, p.1589-1618, 1999.

8 - CORREIA MITD. Avaliação nutricional subjetiva. Rev Bras Nutr Clin 13: 68-73,1998.
9 - BRASIL. Leis, Decretos. Portaria 272 de 08/04/98. Regulamento técnico e requisitos mínimos exigidos para terapia de nutrição parenteral. Diário Oficial da União, Brasília, p.215, 23 abr. 1998.

10 - MARCHINI, J.S.; OKANO, N; CUPO, P; PASSOS, NMRRS; SAKAMOTO, LM \& BATISTA FA. Nutrição parenteral- princípios gerais, formulários de prescrição e monitorização. Medicina, Ribeirão Preto 31:. 62-72, 1998.

11 - SAKAMOTO LM; NAVARRO AM; SUEN VMM; MAXIMO AF \& MARCHINI JS. Administração de cálcio e fósforo na terapia nutricional parenteral. Nutrire, São Paulo 22: 63-71, 2001.

12 - ECHENIQUE M \& CORREIA MITD. Avances en soporte nutricional del pacient crítico. Rev Bras Nutr Clin 13: 221 227,1998 .

13 - CERRA FB ; BENITEZ MR; BLACKBURN GL; IRWIN RS; JEEJEEBHOY K; KATZ DP; PINGLETON SK; POMPOSELLI J; ROMBEAU JL; SHRONTS E; WOLFE RR \& ZALOGA GP. Applied nutrition in ICU pacients. A consensus statement of the American College of Chest Physicians. Chest 111: 769778,1997

14 - WORTHINTON P; GILBERT KA \& WAGNER BA. Parenteral nutrition for the acutely ill. AACN Clin Iss 11: 559-579, 2000 